\title{
Very-high-energy gamma-ray emission from GRB 201216C detected by MAGIC
}

\section{Satoshi Fukami, ${ }^{a}, *$ Alessio Berti, ${ }^{b}$ Serena Loporchio, ${ }^{c}$ Yusuke Suda, ${ }^{d}$ Lara Nava, ${ }^{e}$ Koji Noda, ${ }^{a}$ Željka Bošnjak, ${ }^{f}$ Katsuaki Asano ${ }^{a}$ and Francesco Longo ${ }^{g, h}$ on behalf of the MAGIC Collaboration ${ }^{\dagger}$}

${ }^{a}$ Institute for Cosmic Ray Research, The University of Tokyo, Kashiwanoha 5-1-5, Kashiwa, Japan

${ }^{b}$ Max Planck Institut for Physics, Föhringer Ring 6, Munich, Germany

${ }^{c}$ INFN MAGIC Group: INFN Sezione di Bari and Dipartimento Interateneo di Fisica dell'Università e del Politecnico di Bari, I-70125, Bari, Italy

${ }^{d}$ Physics Program, Graduate School of Advanced Science and Engineering, Hiroshima University, 739-8526 Hiroshima, Japan

${ }^{e}$ National Institute for Astrophysics (INAF), Street number, Merate, Italy

${ }^{f}$ Faculty of Electrical Engineering and Computing, University of Zagreb, Zagreb, Croatia

${ }^{g}$ University of Trieste, Department of Physics, via Valerio 2, Trieste, Italy

${ }^{h}$ INFN, Sezione di Trieste, via Valerio 2, Trieste, Italy

E-mail: fukami@icrr.u-tokyo.ac.jp

Gamma-ray bursts (GRBs) are the most energetic phenomena in the Universe. Many aspects of GRB physics are still under debate, such as the origin of their gamma-ray emission above the $\mathrm{GeV}$ energy range. In 2019, MAGIC detected TeV gamma rays from the long GRB 190114C, whose emission can be well explained by synchrotron-self Compton emission by relativistic electrons. However, it is still unclear whether such a process is common in GRBs, given the reduced number of GRBs detected until now at the very high energies (VHE). GRB 201216C is a long GRB and is the second one detected by MAGIC in this energy range. After receiving the alert provided by Swift-BAT, MAGIC automatically slewed to the GRB position, starting observations 56 seconds after the GRB onset. In the offline analyses of the collected data, we confirmed the detection of gamma-ray emission with a significance above 5 sigma. Following measurements from optical facilities, the redshift of this GRB was estimated to be $z=1.1$. This makes GRB 201216C the most distant object ever detected by ground-based gamma-ray telescopes. In this contribution we will show the analysis results of the MAGIC data, also in comparison with past detected GRBs in the same energy range. Finally, accounting for available multi-wavelength observations, we will comment on the possible origin of the VHE emission detected by MAGIC.

$37^{\text {th }}$ International Cosmic Ray Conference (ICRC 2021)

July 12 th - 23rd, 2021

Online - Berlin, Germany

\footnotetext{
${ }^{*}$ Presenter

${ }^{\dagger}$ a complete list of the MAGIC Collaboration authors can be found at the end of the proceedings
} 


\section{Introduction}

Gamma-ray bursts (GRBs) are the most powerful phenomena in the Universe emitting electromagnetic radiation. Even though more than 50 years have passed since their discovery, many aspects, such as the origin of their prompt emission, the formation of relativistic jets, and the gamma-ray emission mechanism are yet to be properly understood.

The emission from GRBs is classified into two succeeding phases. The initial emission is called prompt emission, which is dominated by soft gamma rays and shows irregular variability of the flux. Depending on the duration of this phase, GRBs are categorized as long or short, a classification which is related to the different progenitor systems producing the GRB. The second phase, which may overlap with the prompt emission, is called afterglow. This phase is characterized by a broadband emission from radio to gamma rays with long lasting duration (from few days to months) and typical temporal decay following a simple power-law.

The current accepted model of the afterglow explains the multiwavelength emission as synchrotron radiation from relativistic electrons, which are accelerated by the forward shock of the jet when it interacts with the interstellar medium and decelerates (e.g. [1]). A contribution from the reverse shock is also evident in many cases (e.g. [2]). As a consequence of this simple model, the multiwavelength spectra and light curves are expected to be described by multiple power-law segments with different indices. Observations are in general agreement with this scenario.

On January 14th, 2019, MAGIC detected for the first time a GRB in the very-high-energy (VHE, roughly $>50 \mathrm{GeV}$ ) range. Hundreds of gamma rays between $300 \mathrm{GeV}$ and $1 \mathrm{TeV}$ were detected from the long-duration GRB 190114C [4]. The emission reached photon energies higher than the maximum energy achievable by synchrotron emission when standard shock acceleration is considered, suggesting the presence of an additional component. The need for an additional spectral component is also supported by the double-bump shaped SED, as constrained by X-ray, LAT and MAGIC simultaneous observations [5]. Even though a synchrotron self-Compton (SSC) emission was found to be able to explain the emission from GRB 190114C in the VHE range, it is not obvious that the SSC emission is energetically relevant among all the GRBs. The H.E.S.S. Collaboration also detected two GRBs in the VHE range several hours after the triggers [6, 7]. For the second one, GRB 190829A, a difficulty in the interpretation of the VHE observations as SSC radiation has been claimed (but see [8]). The data of the GRBs detected by MAGIC and H.E.S.S have variety in many aspects such as observation time after triggers and energetics. More observations of GRBs in the VHE energies are therefore necessary to establish a standard model explaining the origin of the very high energy component.

In this work, we report the MAGIC observation and the analysis result of GRB 201216C, which is the second GRB detected by MAGIC, on December 16th, 2020 [9].

\section{GRB 201216C}

GRB 201216C is a bright long GRB triggered by the Swift-BAT at 23:07:31 UT on December 16th, 2020 (from here on denoted as $T_{0}$ ) [10]. The light curve of the prompt emission consists of multiple peaks between $T_{0}-16 \mathrm{~s}$ and $T_{0}+64 \mathrm{~s}$, and the main peak occurred at $T_{0}+20 \mathrm{~s} \mathrm{[11]}$. $T_{90}$ is $(48 \pm 16) \mathrm{s}$ as measured in the $15-350 \mathrm{keV}$ band by Swift-BAT analysis, and $29.9 \mathrm{~s}$ in the 
50-300 keV band by Fermi-GBM [12]. The time-integrated spectrum between $T_{0}$ and $T_{0}+50 \mathrm{~s}$ is best fitted by a Band function with a peak energy of $(326 \pm 7) \mathrm{keV}$, a low energy index of $-1.06 \pm 0.01$, and a high energy index of $-2.25 \pm 0.03$. The fluence is $(4.5 \pm 0.1) \times 10^{-5} \mathrm{erg} / \mathrm{cm}^{2}$ and $(1.41 \pm 0.06) \times 10^{-4} \mathrm{erg} / \mathrm{cm}^{2}$ in the $15-150 \mathrm{keV}$ and in the $10-1000 \mathrm{keV}$ bands respectively.

The redshift of this GRB is estimated to be $z=1.1$ by the optical observations with the ESO Very Large Telescope (VLT) [13]. The isotropic equivalent energy $E_{\text {iso }}$ in the $10-1000 \mathrm{keV}$ band is $(4.71 \pm 0.16) \times 10^{53} \mathrm{erg}$ calculated from the redshift and the fluence measured by Fermi-GBM.

Optical measurements were performed by several instruments. The ESO VLT detected the afterglow by the observation of one of the telescopes (UT3) with X-shooter spectrograph starting $T_{0}+2.19 \mathrm{~h}$ [14]. The exposures were 120,90,180s in the Sloan $g^{\prime}, r^{\prime}, z^{\prime}$ bands respectively, and the AB magnitude of $r^{\prime}$ band is $21.81 \pm 0.05$. The robotic telescope FRAM-ORM automatically reacted to the alert and observed the GRB from $T_{0}+31.6 \mathrm{~s}$ to around $T_{0}+1000 \mathrm{~s}$ [15]. The duration covered the onset of the afterglow and the power-law temporal decay index is -1.07 estimated by the data combined with the VLT. The Liverpool Telescope (LT) reported detection with a magnitude of 18.38 in the $r^{\prime}$ band at $T_{0}+177 \mathrm{~s}$ [16]. The optical flux shows a peak around this time.

X-ray observations of the late-time afterglow were performed by Swift-XRT, starting at $T_{0}+$ $2966.8 \mathrm{~s}$. The power-law decay index before and after $T_{0}+9078 \mathrm{~s}$ is $-2.09_{-0.10}^{+0.16}$ and $-1.07_{-0.10}^{+0.15}$ respectively [17].

\section{MAGIC observation and analysis}

MAGIC consists of two imaging Cherenkov telescopes with a diameter of $17 \mathrm{~m}$. The MAGIC site is located at an altitude of $2200 \mathrm{~m}$ in the Roque de los Muchachos Observatory on the Canary Island of La Palma in Spain. The target energy range is from $50 \mathrm{GeV}$ to $50 \mathrm{TeV}$ covering a wide range where gamma-ray satellites do not have high sensitivities. MAGIC has a high sensitivity especially for bright transient sources. The integral sensitivity above $105 \mathrm{GeV}$ is $40 \%$ of the flux of Crab Nebula assuming a $0.1 \mathrm{~h}$ observation [18].

Due to the large size of the telescopes, MAGIC can detect dim Cherenkov light, which leads to a relatively low energy threshold compared to other ground-based gamma-ray telescopes. Moreover, the very light carbon fiber structure allows to perform fast rotation of the telescopes so that MAGIC can start observations of transient phenomena with short delay. The follow-up procedure is automatically started as soon as alerts of immediately observable sources such as GRBs are received.

At 23:07:51 UT on December 16th 2020, MAGIC received the alert of GRB 201216C from Swift-BAT and started fast repositioning of the telescopes immediately [9]. MAGIC succeeded to start the observation with a stable trigger rate at $T_{0}+56 \mathrm{~s}$. The observation was performed with the so-called wobble mode, where the telescopes were pointed to a sky position $0.4 \mathrm{deg}$ away from the source position. The MAGIC observation was $2.2 \mathrm{~h}$ of exposure in total. The zenith angle ranged from $37 \mathrm{deg}$ to $68 \mathrm{deg}$ with the energy threshold increasing accordingly. Due to the absence of the moon, the level of the night sky background was low enough to retain dim shower images and therefore obtain a low energy threshold.

The analysis of the data was performed with MAGIC Analysis and Reconstruction Software, MARS [19]. Since gamma rays with energies above a few hundred $\mathrm{GeV}$ are strongly attenuated 
by the extragalactic background light (EBL) given the redshift $z \sim 1.1$ of the source, the energy distribution of the excess counts is concentrated at energies close to the threshold around $100 \mathrm{GeV}$. Therefore, to keep as many low-energy events as possible, we adopted an image cleaning method to extract dimmer Cherenkov showers initiated by gamma rays than the standard method. By using simulated events, we confirmed that more low-energy events survived the event cuts and the sensitivity below $100 \mathrm{GeV}$ was improved with this method.

\section{Results}

The so-called theta2 plot for the first 20 min of the MAGIC observation is shown in Fig. 1. This plot contains the distribution of squared angular distances between the reconstructed positions of gamma-like events and the source position. Such a distribution was also produced around positions without any gamma-ray sources and overlapped to compare it with the one around the source position. The assumed source position is taken from the report by Swift-XRT. The OFF regions are selected so that their centers are at the same distance from the center of the camera as the source position. We selected in total 5 OFF regions and the theta 2 distribution of the OFF regions was obtained by averaging and normalizing the counts. The signal significance is calculated using the formula Eq. 17 in Li\&Ma 1983 [20]. We tried two analysis periods of $20 \mathrm{~min}$ (one data run) and $2.2 \mathrm{~h}$, and obtained $6.0 \sigma$ for the first $20 \mathrm{~min}$. Taking into account a trial number of 2 , the post-trial significance is $5.9 \sigma$, which indicates the clear detection of the VHE emission from the GRB. Fig. 2 shows a significance sky map around the GRB position reported by Swift-XRT. There is a spot above $6 \sigma$ at the position consistent with the GRB within the PSF size.

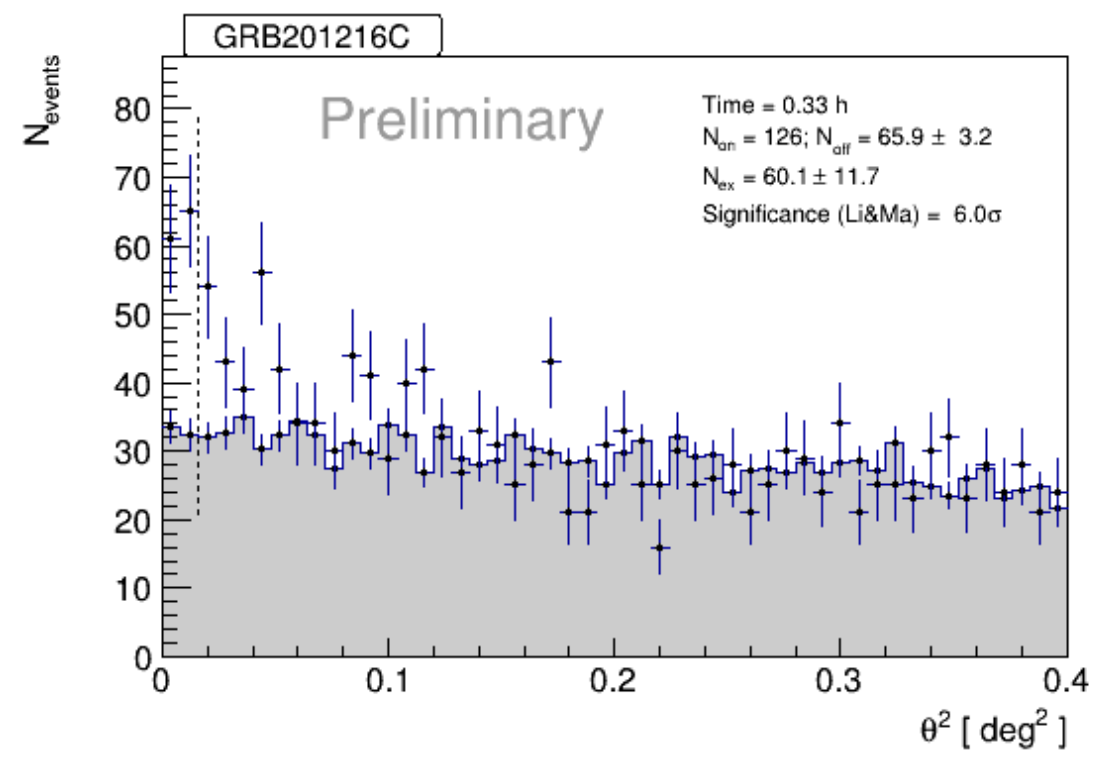

Figure 1: Distribution of angular distances between the reconstructed positions and the source position (theta2 plot). The counts of ON and OFF regions are plotted together. The OFF distribution is filled with grey. The significance is calculated from the counts inside the dotted vertical line with Eq. 17 in LiMa 1983. 


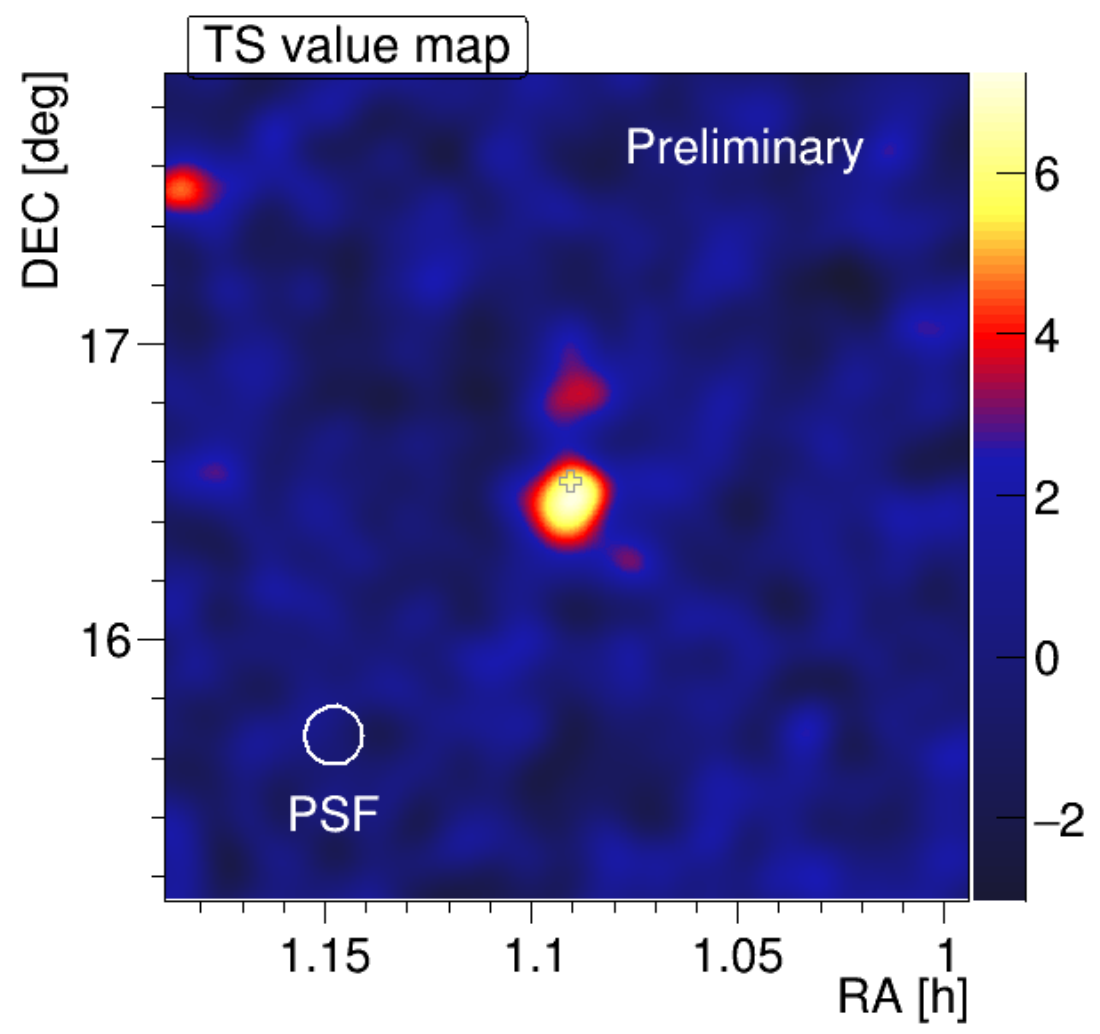

Figure 2: Test statistics map around the position of GRB 201216C reported by Swift-XRT (open cross). The PSF size is shown with the white circle.

We produced a preliminary time-integrated spectrum for the first $20 \mathrm{~min}$. The observed spectrum has a very steep power-law index due to the strong attenuation by EBL from a distance of $z \sim 1.1$, especially for the events with energies higher than a few hundreds of GeV. We then calculated the EBL-corrected spectrum using the EBL model by Dominguez 2011 (hereafter D11 [21]). . The EBL-corrected spectral energy distribution is consistent with a flatter single power-law around $100 \mathrm{GeV}$, too. We have no spectral points above $200 \mathrm{GeV}$ since there are no significant excess counts due to the strong flux attenuation.

Finally, we produced a preliminary photon-flux light curve from $T_{0}+56 \mathrm{~s}$ to $T_{0}+2.2 \mathrm{~h}$. The photon flux is monotonically decaying with time, and the curve is consistent with a power-law. Upper limits are derived for the bins after $T_{0}+50 \mathrm{~min}$ since we do not have significant excess after that time.

\section{Discussion and Conclusions}

MAGIC observed GRB 201216C immediately after receiving the alert and started observations at $T_{0}+56 \mathrm{~s}$. We detected VHE gamma rays with a significance above $5 \sigma$ at the GRB position by the offline analysis of the first 20 min, making the source the second GRB detected by MAGIC. The excess counts are dominated by events around $100 \mathrm{GeV}$, and the observed spectrum is very steep. Both the observed and EBL-corrected spectra are compatible with power-law functions. 
We found that the MAGIC flux decays monotonically with time and is consistent with a powerlaw. The timescale of the MAGIC emission and the temporal behaviour let us conclude that the origin of the VHE radiation is related to the afterglow phase.

The temporal and spectral behaviour of the VHE emission detected from GRB 201216C are qualitatively similar to those of the emission detected from GRB 190114C. However, a detailed study of MWL data is needed to understand if also in this case the emission can be satisfactorily explained in terms of SSC radiation. Currently, the modeling of the MWL observations is being performed to test whether the synchrotron + SSC scenario can explain the multiwavelength data including MAGIC. The results of this study will be the subject of an upcoming paper (MAGIC Collaboration, in preparation).

\section{Acknowledgements}

We acknowledge the support from the agencies and organizations listed here: https:// magic.mpp.mpg.de/acknowledgments_ICRC2021

\section{References}

[1] P. Kumar and B. Zhang. The physics of gamma-ray bursts \& relativistic jets. Physics Reports, 561:1-109, Feb. 2015.

[2] S. Kobayashi. Light curves of gamma-ray burst optical flashes. Astrophysical Journal, 545:807812, Dec. 2000

[3] O. Blanch et al. GCN Circular 29075, Dec. 2020.

[4] V.A. Acciari et al. Teraelectronvolt emission from the $\gamma$-ray burst GRB 190114C. Nature, 575:455-458, Nov. 2019.

[5] V.A. Acciari et al. Observation of inverse Compton emission from a long $\gamma$-ray burst. Nature, 575:459-463, Nov. 2019.

[6] H. Abdalla et al. Revealing x-ray and gamma ray temporal and spectral similarities in the GRB 190829A afterglow. Science, 372:1081-1085, Jun. 2021

[7] H. Abdalla et al. A very-high-energy component deep in the $\gamma$-ray burst afterglow. Nature, 575:464-467, Nov. 2019.

[8] O. S. Salafia et al., Multi-wavelength view of the close-by GRB 190829A sheds light on gamma-ray burst physics, arXiv:2106.07169, 2021.

[9] O. Blanch et al. GCN Circular 29075, Dec. 2020.

[10] A. P. Beardmore et al. GCN Circular 29061, Dec. 2020.

[11] T. N. Ukwatta et al. GCN Circular 29080, Dec. 2020.

[12] C. Malacaria et al. GCN Circular 29073, Dec. 2020. 
[13] J. Vielfaure et al. GCN Circular 29077, Dec. 2020

[14] L. Izzo et al. GCN Circular 29066, Dec. 2020

[15] M. Jelinek et al. GCN Circular 29070, Dec. 2020

[16] M. Shrestha et al. GCN Circular 29085, Dec. 2020

[17] P. A. Evans et al. GCN Circular 29280, Dec. 2020

[18] J. Aleksic et al. The major upgrade of the MAGIC telescopes, Part II: A performance study using observations of the Crab Nebula. Astroparticle Physics, 72:76-94, Jan. 2016.

[19] R. Zanin et al. Proceedings, 33rd International Cosmic Ray Conference (ICRC2013), p2937 July 2013

[20] T. Li and Y. Ma. Analysis methods for results in gamma-ray astronomy. Astrophysical Journal, 272:317-324, Sep. 1983

[21] A. Dominguez et al. Extragalactic background light inferred from AEGIS galaxy-SED-type fractions. Monthly Notices of the Royal Astronomical Society, 410:2556-2578, Feb. 2011.

\section{The MAGIC Collaboration}

V. A. Acciari ${ }^{1}$, S. Ansoldi ${ }^{2,41}$, L. A. Antonelli ${ }^{3}$, A. Arbet Engels ${ }^{4}$, M. Artero ${ }^{5}$, K. Asano ${ }^{6}$, D. Baack ${ }^{7}$, A. Babić ${ }^{8}$, A. Baquero ${ }^{9}$, U. Barres de Almeida ${ }^{10}$, J. A. Barrio ${ }^{9}$, I. Batković ${ }^{11}$, J. Becerra González ${ }^{1}$, W. Bednarek ${ }^{12}$, L. Bellizzi ${ }^{13}$, E. Bernardini ${ }^{14}$, M. Bernardos ${ }^{11}$, A. Berti ${ }^{15}$, J. Besenrieder ${ }^{15}$, W. Bhattacharyya ${ }^{14}$, C. Bigongiari ${ }^{3}$, A. Biland ${ }^{4}$, O. Blanch ${ }^{5}$, H. Bökenkamp ${ }^{7}$, G. Bonnoli ${ }^{16}$, Ž. Bošnjak ${ }^{8}$, G. Busetto ${ }^{11}$, R. Carosi ${ }^{17}$, G. Ceribella ${ }^{15}$, M. Cerruti ${ }^{18}$, Y. Chai ${ }^{15}$, A. Chilingarian ${ }^{19}$, S. Cikota $^{8}$, S. M. Colak ${ }^{5}$, E. Colombo ${ }^{1}$, J. L. Contreras ${ }^{9}$, J. Cortina ${ }^{20}$, S. Covino ${ }^{3}$, G. D’Amico ${ }^{15,42}$, V. D’Elia ${ }^{3}$, P. Da Vela ${ }^{17,43}$, F. Dazzi ${ }^{3}$, A. De Angelis ${ }^{11}$, B. De Lotto ${ }^{2}$, M. Delfino ${ }^{5,44}$, J. Delgado ${ }^{5,44}$, C. Delgado Mendez $^{20}$, D. Depaoli ${ }^{21}$, F. Di Pierro ${ }^{21}$, L. Di Venere ${ }^{22}$, E. Do Souto Espiñeira ${ }^{5}$, D. Dominis Prester $^{23}$, A. Donini ${ }^{2}$, D. Dorner ${ }^{24}$, M. Doro ${ }^{11}$, D. Elsaesser ${ }^{7}$, V. Fallah Ramazani ${ }^{25,45}$, A. Fattorini ${ }^{7}$, M. V. Fonseca ${ }^{9}$, L. Font ${ }^{26}$, C. Fruck ${ }^{15}$, S. Fukami ${ }^{6}$, Y. Fukazawa ${ }^{27}$, R. J. García López ${ }^{1}$, M. Garczarczyk ${ }^{14}$, S. Gasparyan ${ }^{28}$, M. Gaug ${ }^{26}$, N. Giglietto ${ }^{22}$, F. Giordano ${ }^{22}$, P. Gliwny ${ }^{12}$, N. Godinović ${ }^{29}$, J. G. Green ${ }^{3}$, D. Green ${ }^{15}$, D. Hadasch ${ }^{6}$, A. Hahn ${ }^{15}$, L. Heckmann ${ }^{15}$, J. Herrera ${ }^{1}$,

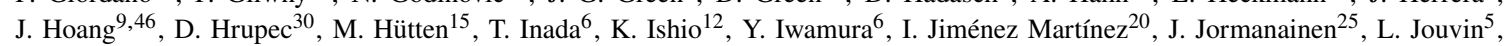
M. Karjalainen ${ }^{1}$, D. Kerszberg ${ }^{5}$, Y. Kobayashi ${ }^{6}$, H. Kubo ${ }^{31}$, J. Kushida ${ }^{32}$, A. Lamastra ${ }^{3}$, D. Lelas ${ }^{29}$, F. Leone ${ }^{3}$, E. Lindfors ${ }^{25}$, L. Linhoff ${ }^{7}$, S. Lombardi ${ }^{3}$, F. Longo ${ }^{2,47}$, R. López-Coto ${ }^{11}$, M. López-Moya ${ }^{9}$, A. López-Oramas ${ }^{1}$, S. Loporchio ${ }^{22}$, B. Machado de Oliveira Fraga ${ }^{10}$, C. Maggio ${ }^{26}$, P. Majumdar ${ }^{33}$, M. Makariev ${ }^{34}$, M. Mallamaci ${ }^{11}$, G. Maneva ${ }^{34}$, M. Manganaro ${ }^{23}$, K. Mannheim ${ }^{24}$, L. Maraschi ${ }^{3}$, M. Mariotti ${ }^{11}$, M. Martínez ${ }^{5}$, D. Mazin ${ }^{6,15}$, S. Menchiari ${ }^{13}$, S. Mender ${ }^{7}$, S. Mićanović23, D. Miceli ${ }^{2,49}$, T. Miener ${ }^{9}$, J. M. Miranda ${ }^{13}$, R. Mirzoyan ${ }^{15}$, E. Molina ${ }^{18}$, A. Moralejo ${ }^{5}$, D. Morcuende ${ }^{9}$, V. Moreno ${ }^{26}$, E. Moretti ${ }^{5}$, T. Nakamori ${ }^{35}$, L. Nava ${ }^{3}$, V. Neustroev ${ }^{36}$, C. Nigro ${ }^{5}$, K. Nilsson ${ }^{25}$, K. Nishijima ${ }^{32}$, K. Noda ${ }^{6}$, S. Nozaki ${ }^{31}$, Y. Ohtani ${ }^{6}$, T. Oka ${ }^{31}$, J. Otero-Santos ${ }^{1}$, S. Paiano ${ }^{3}$, M. Palatiello ${ }^{2}$, D. Paneque ${ }^{15}$, R. Paoletti ${ }^{13}$, J. M. Paredes ${ }^{18}$, L. Pavletić ${ }^{23}$, P. Peñil ${ }^{9}$, M. Persic ${ }^{2,50}$, M. Pihet ${ }^{15}$, P. G. Prada Moroni ${ }^{17}$, E. Prandini ${ }^{11}$, C. Priyadarshi ${ }^{5}$, I. Puljak ${ }^{29}$, W. Rhode ${ }^{7}$, M. Ribó ${ }^{18}$, J. Rico ${ }^{5}$, C. Righi ${ }^{3}$, A. Rugliancich ${ }^{17}$, N. Sahakyan ${ }^{28}$, T. Saito ${ }^{6}$, S. Sakurai ${ }^{6}$, K. Satalecka ${ }^{14}$, F. G. Saturni ${ }^{3}$, B. Schleicher ${ }^{24}$, K. Schmidt $^{7}$, T. Schweizer ${ }^{15}$, J. Sitarek ${ }^{12}$, I. Šnidaric ${ }^{37}$, D. Sobczynska ${ }^{12}$, A. Spolon ${ }^{11}$, A. Stamerra ${ }^{3}$, J. Strišković ${ }^{30}$, D. Strom ${ }^{15}$, M. Strzys ${ }^{6}$, Y. Suda ${ }^{27}$, T. Surićc ${ }^{37}$, M. Takahashi ${ }^{6}$, R. Takeishi' ${ }^{6}$, F. Tavecchio ${ }^{3}$,

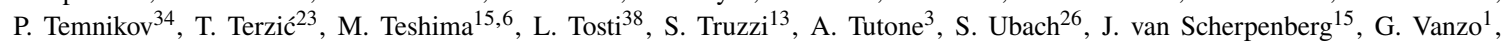
M. Vazquez Acosta ${ }^{1}$, S. Ventura ${ }^{13}$, V. Verguilov ${ }^{34}$, C. F. Vigorito ${ }^{21}$, V. Vitale ${ }^{39}$, I. Vovk $^{6}$, M. Will $^{15}$, C. Wunderlich ${ }^{13}$, T. Yamamoto ${ }^{40}$, and D. Zaric ${ }^{29}$

${ }^{1}$ Instituto de Astrofísica de Canarias and Dpto. de Astrofísica, Universidad de La Laguna, E-38200, La Laguna, Tenerife, Spain ${ }^{2}$ Università di Udine and INFN Trieste, I-33100 Udine, Italy ${ }^{3}$ National Institute for Astrophysics (INAF), I-00136 Rome, Italy ${ }^{4}$ ETH Zürich, CH-8093 Zürich, Switzerland ${ }^{5}$ Institut de Física d'Altes Energies (IFAE), The Barcelona Institute of Science and Technology (BIST), E-08193 Bellaterra (Barcelona), Spain ${ }^{6}$ Japanese MAGIC Group: Institute for Cosmic Ray Research (ICRR), The University of Tokyo, Kashiwa, 277-8582 Chiba, Japan ${ }^{7}$ Technische Universität Dortmund, D-44221 Dortmund, Germany ${ }^{8}$ Croatian MAGIC Group: University of Zagreb, Faculty of Electrical Engineering and Computing (FER), 10000 Zagreb, Croatia ${ }^{9}$ IPARCOS Institute and EMFTEL Department, Universidad Complutense de Madrid, E-28040 Madrid, Spain ${ }^{10}$ Centro Brasileiro de Pesquisas Físicas (CBPF), 
22290-180 URCA, Rio de Janeiro (RJ), Brazil ${ }^{11}$ Università di Padova and INFN, I-35131 Padova, Italy ${ }^{12}$ University of Lodz, Faculty of Physics and Applied Informatics, Department of Astrophysics, 90-236 Lodz, Poland ${ }^{13}$ Università di Siena and INFN Pisa, I-53100 Siena, Italy ${ }^{14}$ Deutsches Elektronen-Synchrotron (DESY), D-15738 Zeuthen, Germany ${ }^{15}$ Max-Planck-Institut für Physik, D-80805 München, Germany ${ }^{16}$ Instituto de Astrofísica de Andalucía-CSIC, Glorieta de la Astronomía s/n, 18008, Granada, Spain ${ }^{17}$ Università di Pisa and INFN Pisa, I-56126 Pisa, Italy ${ }^{18}$ Universitat de Barcelona, ICCUB, IEEC-UB, E-08028 Barcelona, Spain ${ }^{19}$ Armenian MAGIC Group: A. Alikhanyan National Science Laboratory, 0036 Yerevan, Armenia ${ }^{20}$ Centro de Investigaciones Energéticas, Medioambientales y Tecnológicas, E-28040 Madrid, Spain ${ }^{21}$ INFN MAGIC Group: INFN Sezione di Torino and Università degli Studi di Torino, I-10125 Torino, Italy ${ }^{22}$ INFN MAGIC Group: INFN Sezione di Bari and Dipartimento Interateneo di Fisica dell'Università e del Politecnico di Bari, I-70125 Bari, Italy ${ }^{23}$ Croatian MAGIC Group: University of Rijeka, Department of Physics, 51000 Rijeka, Croatia ${ }^{24}$ Universität Würzburg, D-97074 Würzburg, Germany ${ }^{25}$ Finnish MAGIC Group: Finnish Centre for Astronomy with ESO, University of Turku, FI-20014 Turku, Finland ${ }^{26}$ Departament de Física, and CERES-IEEC, Universitat Autònoma de Barcelona, E-08193 Bellaterra, Spain ${ }^{27}$ Japanese MAGIC Group: Physics Program, Graduate School of Advanced Science and Engineering, Hiroshima University, 739-8526 Hiroshima, Japan ${ }^{28}$ Armenian MAGIC Group: ICRANet-Armenia at NAS RA, 0019 Yerevan, Armenia ${ }^{29}$ Croatian MAGIC Group: University of Split, Faculty of Electrical Engineering, Mechanical Engineering and Naval Architecture (FESB), 21000 Split, Croatia ${ }^{30}$ Croatian MAGIC Group: Josip Juraj Strossmayer University of Osijek, Department of Physics, 31000 Osijek, Croatia ${ }^{31}$ Japanese MAGIC Group: Department of Physics, Kyoto University, 606-8502 Kyoto, Japan ${ }^{32}$ Japanese MAGIC Group: Department of Physics, Tokai University, Hiratsuka, 259-1292 Kanagawa, Japan ${ }^{33}$ Saha Institute of Nuclear Physics, HBNI, 1/AF Bidhannagar, Salt Lake, Sector-1, Kolkata 700064, India ${ }^{34}$ Inst. for Nucl. Research and Nucl. Energy, Bulgarian Academy of Sciences, BG-1784 Sofia, Bulgaria ${ }^{35}$ Japanese MAGIC Group: Department of Physics, Yamagata University, Yamagata 990-8560, Japan ${ }^{36}$ Finnish MAGIC Group: Astronomy Research Unit, University of Oulu, FI-90014 Oulu, Finland ${ }^{37}$ Croatian MAGIC Group: Ruđer Bošković Institute, 10000 Zagreb, Croatia ${ }^{38}$ INFN MAGIC Group: INFN Sezione di Perugia, I-06123 Perugia, Italy ${ }^{39}$ INFN MAGIC Group: INFN Roma Tor Vergata, I-00133 Roma, Italy ${ }^{40}$ Japanese MAGIC Group: Department of Physics, Konan University, Kobe, Hyogo 6588501, Japan ${ }^{41}$ also at International Center for Relativistic Astrophysics (ICRA), Rome, Italy ${ }^{42}$ now at Department for Physics and Technology, University of Bergen, NO-5020, Norway ${ }^{43}$ now at University of Innsbruck ${ }^{44}$ also at Port d'Informació Científica (PIC), E-08193 Bellaterra (Barcelona), Spain ${ }^{45}$ now at Ruhr-Universität Bochum, Fakultät für Physik und Astronomie, Astronomisches Institut (AIRUB), 44801 Bochum, Germany ${ }^{46}$ now at Department of Astronomy, University of California Berkeley, Berkeley CA 9472047 also at Dipartimento di Fisica, Università di Trieste, I-34127 Trieste, Italy 49 now at Laboratoire d'Annecy de Physique des Particules (LAPP), CNRS-IN2P3, 74941 Annecy Cedex, France ${ }^{50}$ also at INAF Trieste and Dept. of Physics and Astronomy, University of Bologna, Bologna, Italy 